\title{
Can we help in changing the future of Italian health care?
}

Mario Di Napoli ${ }^{1}$

UOC Neurologia. Ospedale Generale Provinciale San Camillo de' Lellis, Rieti

The retirement age of the baby boom generation is almost out-door. The proportion of ageing people will grow rapidly in the near future [1-3]. There will be fewer people to pay for the health and social care of the quickly aging population; furthermore, advancements in medical science will raise a new bar for quality in terms of the outcomes achieved in the treatment of illness [3]. An ageing population and the rising public expectations will produce an increase in costs and will impede timely access to care, thus jeopardising sustainability.

The Italian National Health system is an example of a collapsing system: the ageing Italian workforce affects not only patient demographics but also the availability of clinicians. The upcoming wave of retiring health care professionals will occur in the exact moment when they will be more necessary. To overcome these challenges, our health care system will have to use its resources more effectively. Access and equity remain essential characteristics of our health care system, but are insufficient goals in terms of improving quality and achieving financial sustainability. Decades of reforms have not solved the problem, and current solutions, such as strict cost controls, empowering consumers, or betting on internet technology represent simplistic solutions. This fact creates an inefficient monopoly with irresistible pressures to exert bargaining power, limits services, and attempts to micromanage care delivery. It will only make things getting worse by exacerbating the zero-sum competition present in the current system [4]. The present point of view considers the treatments and the health service performances as the main aim and the final product of an efficient national health system. This is the price of common wishful thinking about our health care service performances. The belief that treatments are always effective, and that prevention always works is profoundly seductive to patients, who wish to benefit; to physicians, who want to be helpful; to politicians, who want the health service to be cost effective; and to the whole medical industrial complex, whose technologies and pharmaceuticals drive enormous profits [5]. An example of this overoptimistic point of view is stroke medicine, where the benefits of treatment are exaggerated and wishful thinking often trumps the published evidence. The Royal College of Physicians' press release that accompanied its 2010 stroke audit report in England is a recent pertinent example [6]. The concentration on the hyper-acute care [7] along with wishful thinking [6] are having and have had a high price in stroke medicine, without a true everyday clinical benefit $[8,9]$. The futility 
of this approach is due to a fragmented intervention instead of an integrated approach. Clinical care delivery models differ between different diseases. Effective care processes will reflect the predisposing factors, the cause (aetiology), course and consequences of a particular disease, and the available therapy options and their costs. Depending on the nature of the disease, care may be delivered most appropriately in primary, emergency or palliative care settings; it may be focused to different extents on prevention, management, or cure; and it may be characterised by remission states with flare-ups (Figure 1).

Value for the patient involves the full cycle of care, not just the outcome of a single intervention. The "good" or product is health, not treatment. Better health is inherently less expensive than poor health and a higher quality care is often less costly than a low quality one. It was assumed that the quality of health care was good enough, and seen the problem as cost. However, obtaining a better health status is different from buying a car, where a sporting car is inherently more costly than a city car. Furthermore, the experience of the last decades has shown that the more the focus is on driving down costs, the more costs tend to increase. This is because the efforts to control costs often degenerate to cost shifting and eliminating discretionary, but this monitoring services-based approach has introduced only major inefficiencies in the system. In the long run, when the interventions on the health status of a population should be evaluated, the value of these interventions is obviously eroded.

The best way to reduce costs is to drive improvements in quality, but a true quality as measured by results. There is consistent evidence that better quality care leads to an improved efficiency. So, perhaps more than in any other sectors, better quality inherently reduces costs. Prevention and disease management cost less than acute treatment and rehabilitation. Treatment earlier in the causal chain is less costly. Getting the diagnosis right is more efficient than failed or unnecessary treatment. Fewer mistakes and complications cost less. Less invasive treatments enable less expensive recovery. Getting the right form of treatment to the right patients reduces the costs of failed or ineffective therapies. Faster recovery is less expensive than convalescence. Less disability means less long-term care.

At the present point, the system must be restructured to radically improve its value. Otherwise, increasing amounts of rationing and administrative control of health care are inevitable. The only way to truly reform the health care system is a value-based competition on results, which is defined as the health outcomes achieved per Euro spent [4]. In synthesis, the goal is to increase value and not just contain costs. Value is measured by the overall health outcome achieved relative to the total costs of care over

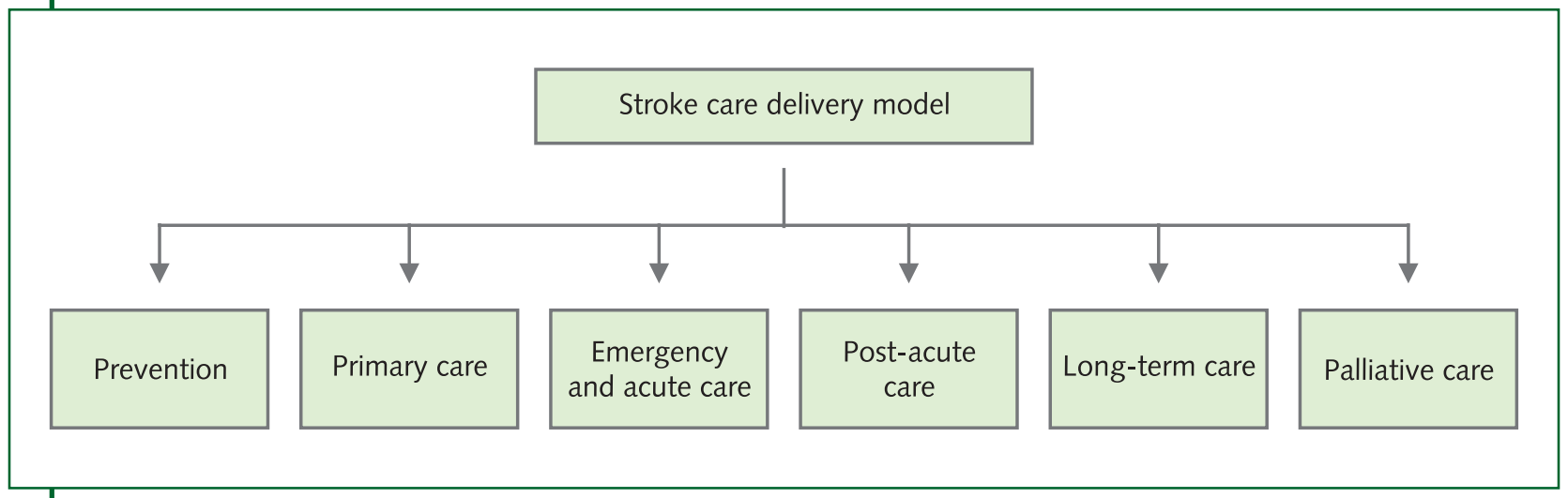

Figure 1. Continuum of health care delivery. Figure shows the main types of health care delivery settings. These are not mutually exclusive. For example, care for stroke spans several settings emergency, acute and post-acute. The organisation and delivery of health care services are complex concepts that are challenging to define. The delivery system includes a variety of personnel, processes and infrastructure elements 
the full cycle of the patient's illnesses. Value-based health care delivery seeks to minimise the overall costs of care, not just focus on minimising the cost of individual services or interventions. Value-based care delivery spends more on appropriate services in order to save through early intervention, reducing mistakes, minimising complications, and forestalling disease recurrences.

However, to achieve this goal, the physician's understanding of this process must be changed. Needs for health care are determined by the patient's medical condition. Care for a medical condition must integrate not only the activities directly related to the disease, but also to the related conditions, and defined for patient groups with similar needs [10]. From this point of view, patient populations requiring different bundles of primary and preventive care services might include, for example, healthy children, healthy adults, patients with a single chronic disease, frail elderly people, and patients with multiple chronic conditions. Each patient group has unique needs and requires inherently different primary care services which are best delivered by different teams, and potentially in different settings and facilities. Care for a medical condition usually involves multiple specialties and numerous interventions. Value for the patient is created not by each intervention or specialty, but by the combined efforts of all of them. Patient outcomes will depend on a sequence of interventions often involving different sites and types of care - outpatient care, inpatient care, office visits, tests, rehabilitation, counselling, medications, procedures, and so on. The benefits of each intervention for ultimate outcomes will depend on the effectiveness of other interventions throughout the care cycle. Because care activities are interdependent, value for patients is often revealed only over time and manifested in longer-term outcomes such as sustainable recovery, need for ongoing interventions, or occurrences of treatmentinduced illnesses. The only way to accurately measure the value of an effective health intervention is to track individual patient outcomes and costs longitudinally over the full care cycle.

This is the integrated point of view of Review in Health Care: showing the future avenues in health care and suggesting how they can help in changing the health care. This mission is fundamental for a Journal as $R H C$ which has the high-flying mission of improving the health care quality because, quoting Adam Smith: «We trust our health to the physician [...]. Such confidence could not safely be reposed in people of a very mean or low condition. Their reward must be such, therefore, as may give them that rank in the society which so important a trust requires. The long time and great expense which must be laid out in their education, when combined with this circumstance, necessarily enhance still further the price of their labour». We want to create a system that encourages innovation and drives towards excellence and efficiency over time.

Let's get started!

\section{References}

1. Rogerson PA, Kim D. Population distribution and redistribution of the baby-boom cohort in the United States: recent trends and implications. Proc Natl Acad Sci USA 2005; 102: 15319-24

2. Parker MG, Thorslund M. Health trends in the elderly population: getting better and getting worse. Gerontologist 2007; 47: 150-8

3. Smith K, Toder E, Iams H. Lifetime distributional effects of Social Security retirement benefits. Soc Secur Bull 2003; 65: 33-61

4. Porter ME. What is value in health care? N Engl J Med 2010; 363: 2477-81

5. Heath I. The price of wishful thinking. BMJ 2010; 341

6. Royal College of Physicians. Better community care needed to help stroke victims recover. Perss release, 19 August 2010

7. Fisher M. Developing and implementing future stroke therapies: the potential of telemedicine. Ann Neurol 2005; 58: 666-71 
8. Price CI, Clement F, Gray J, Donaldson C, Ford GA. Systematic review of stroke thrombolysis service configuration. Expert Rev Neurother 2009; 9: 211-33

9. National Institute for Health and Clinical Excellence. NICE cost impact and commissioning assessment: quality standard for stroke. NICE, 2010. Available at: http:/www.nice.org.uk/ media/87D/33/StrokeCostingCommissioningAssessment.pd

10. Krumholz HM, Keenan PS, Brush JE Jr, Bufalino VJ, Chernew ME, Epstein AJ, et al; American Heart Association Interdisciplinary Council on Quality of Care and Outcomes Research; American College of Cardiology Foundation. Standards for measures used for public reporting of efficiency in health care: a scientific statement from the American Heart Association Interdisciplinary Council on Quality of Care and Outcomes research and the American College of Cardiology Foundation. J Am Coll Cardiol 2008; 52: 1518-26

\section{Featured in this Issues}

The intracerebral haemorrhage associated to oral anticoagulant therapy: the practical management of urgent reversal therapy

- Medical area. Internal medicine, emergency medicine, cardiology, geriatrics, neurology, neurosurgery, neuroradiology, haemostasis and thrombosis.

- Why is a review on this topic needed? The great diffusion of oral anticoagulant therapy (OAT), especially in elderly patients, and the consequent major haemorrhages, often fatal, makes this review of the literature essential to widespread the indications for the correct management of the urgent OAT reversal. In particularly, this review highlights the drugs currently available in Italy, their advantages and disadvantages, their correct usage and controindications.

- Key message. Strategies for urgent oral anticoagulant therapy reversal remain underused but they are safe and efficacious and could reduce mortality of OAT related intracerebral haemorrhage.

Smoking cessation interventions in nurses and other health care workers

- Medical area. Public health and prevention.

- Why is a review on this topic needed? Tobacco smoking is the leading preventable cause of the most important diseases and the treatment of tobacco-related diseases makes up an economic burden to the health care system as well as to society. Health care professionals have an important role to play both as advisers, influencing smoking cessation, and as role models.

- Key message. There is evidence that smoking cessation interventions among health care workers can be effective. This is of particular interest both for reducing tobacco smoking prevalence among this type of workers and for helping them to be useful model for the general population.

Chronic obstructive pulmonary disease and comorbidity: possible implications in the disease management

- Medical area. Internal medicine, geriatrics, cardiology, general medicine, respiratory medicine.

- Why is a review on this topic needed? Chronic obstructive pulmonary disease (COPD) is becoming the first cause of pulmonary disability and death. Because of the increase in the mean age of the population, COPD is frequently associated with important comorbidities that require medical attention.

- Key message. An interdisciplinary co-operation between all the clinicians involved in the management of the disease could be useful in promoting specific strategies able to affect the several pulmonary and extra-pulmonary components of the disease. New pharmacologic options active on both pulmonary and extra-pulmonary inflammation might be useful in the future. 
Perioperative intensive care in patients with brain tumours

- Medical area. Neurosurgery, neurology, intensive care.

- Why is a review on this topic needed? The surgery of brain tumours is not free from complications. Preoperative evaluation aims at minimising the risks; nevertheless this evaluation has not yet been defined and is not based on a strong evidence.

- Key message. Neuroimaging techniques and the progresses of microsurgery, neuromonitoring and dedicated intensive care units contributed in recent years to improving the results of neurosurgery. Perioperative multidisciplinary management is essential in improving the outcomes. 